\title{
On Analytical Solution of a Plasma over a Moving Rigid Plate under the Influence of Non-linear Non-uniform Applied External Magnetic Field.
}

\author{
Taha Zakaraia Abdel Wahid A and Adel M. Morad A, B \\ ${ }^{\text {A }}$ Department of Mathematics and Computer Science, Faculty of Science, Menoufia University, Shebin El-Kom \\ 32511, Egypt \\ ${ }^{B}$ Department of Computational Mathematics and Mathematical Physics, Institute of Mathematics, Mechanics and \\ Computer Science, Southern Federal University, Rostov on Don 344090, Russia \\ Emails: taha_zakaraia@yahoo.com; dr_adel_morad@yahoo.com
}

\begin{abstract}
In this study, we mainly focus on the behavior of an electron gas produced by argon plasma that is bounded by a moving rigid flat plate. The effects of an external magnetic field on the electrons collected with each other, with positive ions, and with neutral atoms in the plasma fluid are investigated. We used the BGK model of the Boltzmann kinetic equation, which is fundamental in the study of the gas dynamics from the continuum flow to the free-molecular regimes with Maxwellian velocity distribution functions of the various species. An exact analytical solution of the model equations for the unsteady flow is given using the moment and the traveling wave methods. The behavior of the mean velocity of electrons is illustrated, which compatible with the variation of the shear stress, viscosity coefficient, and the initial and boundary conditions. Besides, the thermodynamic prediction is investigated by applying the principles of irreversible thermodynamics and Gibbs formula. It is found that the thermodynamic variables verify the Boltzmann's H-theorem, the principle of Le Chatelier, and the Thermodynamics Second Law for the non-equilibrium processes of the system. Qualitative agreements with previous related papers are introducing. 3-Dimensional graphics for the calculating variables are offering, and their behavior is deeply discussed.
\end{abstract}

PACS: 52.30.-q, 71.10.Ca, 52.35.Sb, 52.25.Kn.

Keywords: Plasmas; Fluid mechanics; Compressible flow; Statistical mechanics theorems; Irreversible thermodynamics; Maxwell-Boltzmann distribution; Electron gas; Magnetic fields; Laws of thermodynamics; Viscosity; Boltzmann kinetic equation.

\section{Introduction}

From basic research in plasma science to manufacturing, rapid developments in that field have often been preceded by revolutions in new technologies, such as low-temperature plasmas, or novel applications, such as the field of plasma medicine, plasma biological systems, and microelectronics [1]. For example, Miller et al. [2] have used the non-equilibrium plasma to induce immunogenic cell death in tumors as a therapeutic way for diseases of body systems exposed to the plasma. Plasma-surface interactions have gained immense interest in the last few decades in the context of promising industrial applications in the world's global commercial products, manufacturing processes, and, more recently, microelectronics, medical, and biotechnologies, which are discussed elsewhere in [3].

It is well known that there are two kinetic methods for mathematical modeling of the behavior of a particle population. These methods deal with a system of differential equations that describe the variations of the phase space distribution function $\varphi(t, \mathbf{r}, \mathbf{v})$. In the case of collisionless plasma, 
this model equation is the "Vlasov-equation" [4]. In the collisional case, the Boltzmann's kinetic equation can be used to describe the motion of collisional plasma, where the microscopic effects are considered. To treat the difficulty of studying the collision term in Boltzmann's kinetic equation, we should use approximate modeling. One of the essential approximate models is the Bhatnagar-Gross-Krook model (BGK), which is used to save the computational cost of the collision term of Boltzmann's kinetic equation [5, 6-7].

After the pioneering work by Chapman [8], Enskog [9], and the more recent modification of Chapman-Enskog method [10], many theoretical and numerical contributions interest in developing the methods to obtain reasonable solutions to the Boltzmann equation [11,12]. Furthermore, researchers have been focused on increasing their understanding of gas-kinetic flow theory based on the Boltzmann kinetic equation in comparison with the macroscopic description found by following the Navier-Stokes (NS) equations, for example, many approximations have been suggested to solve Boltzmann's equation based on the method of moment, such as Grad's moment method, Krook's model, and Lee's method [13, 14-22]. The first efforts to model the solution of Boltzmann's equation used Chapman-Enskog procedure simplifications (see [10]). Recent progress has allowed a more suitable method which is Grad's method and method of moments with discontinuous distribution function [11].

In the Chapman-Enskog method, transport equations are constructed at successive levels of approximation by expanding the distribution function for the parameters around the equilibrium distribution function (Maxwellian) and the matrix elements of the expansion tensors, while in Grad's method the transport equations for macroscopic molecular averages are carried out by taking velocity moments of the distribution function which is approximated by an expansion in orthonormal polynomials [23]. Alternatively, Kremer obtained the so-called Chapman-EnskogGrad combined method for constitutive equations by developing the features of the ChapmanEnskog technique and Grad's moment method [24].

Many recent studies on the possibility of the application of fluid models for incompressible viscous continuum fluids are well introduced for the collisional plasma. However, in the case of collisionless plasma, we cannot use these models. However, the magnetohydrodynamics (MHD) models have been used to explain the collisionless plasma and to identify the macroscopic parameters, such as fluid velocity, density, and pressure [4].

Interaction between moving plasmas and solid surfaces in modern technological applications has a rich history throughout the history of plasma technologies. For example, Chang and Chang [25] have studied Rayleigh's problem for plasma boundary layer flow in an incompressible viscous case under the effect of an applied magnetic field. Abourabia and Tolba [26] investigated the behavior of a rarefied electron gas generated from noble gases using the method of moments in case of a discontinuous distribution function. They obtained an approximate solution for the mean flow velocity and shear stress. Furthermore, they considered that the immobile ions are kept as uniform and neutralizing background. In our work, we obtain an exact solution for the model equations and study the problem completely, considering the influence of electron-ion and electron -atom collisions in the Boltzmann equation collision term. The Rayleigh flow characteristics of a rarefied electron gas generated by neutral atoms have been identified and explained in [21-22, 27].

Further, they explained the behavior of the gas system but with an approximate solution and an inaccurate collision frequency formula. Abdel Wahid [20] presented an exact solution of Boltzmann kinetic equation with 4-collision frequency terms. In this study, we treat the complete collision frequencies of the Boltzmann kinetic equation and introduce a certain form of the model solution to avoid the discontinuity of the solutions. Also, we deal with the total values of variables 
without any cut-off caused by the small parameters' method implemented in $[27,28]$. In the present paper, we had a significant advantage that we have taken into consideration the current displacement terms in the Maxwell equations, which are ignored in all mentioned papers previously, see $[17,18,20,26,28]$, for simplifying the differential equations system to can be solved more easily. Nevertheless, we did not ignore this term here because of the applied unsteady external magnetic field that given this term great importance in our calculations, and it cannot be ignored at all.

The benefit of treating the Boltzmann kinetic equation enables us to consider the concepts of the linear non-equilibrium irreversible thermodynamics [6]. Besides, the using of Boltzmann's Htheorem and Gibbs formulae with the distribution functions give researchers the possibility to determine the entropy, entropy production, thermodynamic forces, and kinetic coefficient of the plasma systems and illustrate the physical interpretation of the non-equilibrium thermodynamic properties of the whole system [29-30].

\section{The Physical Situation and Mathematical Formulation}

Assuming the upper half of the space $\mathrm{y} \geq 0$ is bounded by an illimitable plane plate at $\mathrm{y}=0$. Space is filled with a plasma gas under the influence of an unsteady non-uniform external magnetic field $\left(\boldsymbol{B}_{z E}\right.$ ) normal to the direction of the flow. The plasma gas is initially in equilibrium. Considering this plasma in an applied magnetic field, we utilize the customary geometry, as the electric field direction is along the $\mathrm{x}$-axis, and the applied magnetic field direction is along the $\mathrm{z}$ axis. The plane plate moves suddenly in its plane with a damping velocity $\left(V_{0} e^{-\alpha t}\right)$ along the $\mathrm{x}-$ axis.

Moreover, the plane plate is considered an insulator, uncharged, and impermeable. The system components (the plane plate +electrons + positive ions + neutral atoms) are kept at a constant temperature. We treated a frequency regime such that we can neglect ion currents compared to electron currents. Therefore, one can neglect the motion of ions and pear our attention to electron motion.

The Lorentz force $\stackrel{\perp}{F}_{e}$ acting on each electron; be acquired by:

(1) $\stackrel{\mathrm{r}}{F_{e}}=-e \stackrel{\mathrm{r}}{E}-\frac{e}{c}(\stackrel{\mathrm{r}}{\xi} \wedge \stackrel{\mathrm{r}}{B})$, here $\stackrel{\stackrel{\mathbf{r}}{\boldsymbol{B}}}{=}=B_{z} \stackrel{\mathbf{I}}{\boldsymbol{k}}=\left(B_{z E}+B_{z I}\right) \stackrel{\mathbf{l}}{k}$, as $B_{z E}=B_{0} e^{\phi(k y-\omega t)}$

Here $B_{z E}$ is the applied unsteady non-uniform external magnetic field and $B_{z I}$ is the induced magnetic field. They are functions of $y$, and $t$ as $\phi, \omega$, and $\kappa$ are constants. Here

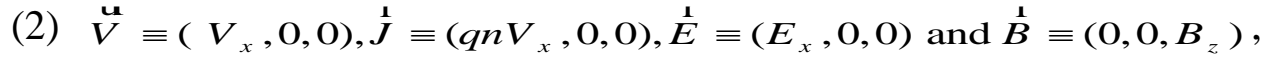

as $V_{x}, E_{x}, B_{z}$, and $J_{x}$ are functions of $y$ and $t$. In particular, this preference satisfies Maxwell's equations. In our model, the distribution function $\varphi_{e}(\stackrel{r}{r}, \stackrel{1}{\xi}, t)$ of the electrons in the plasma gas can be acquired by the Boltzmann kinetic equation that can be written in the BGK model in the form $[5,6]$ :

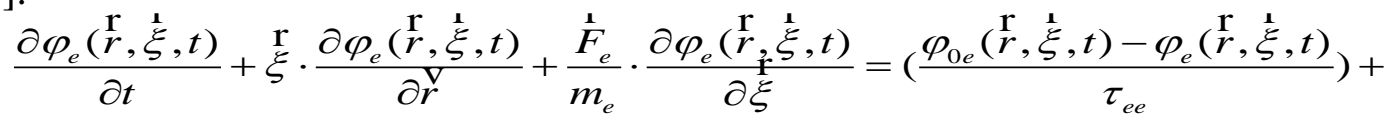

$$
\begin{aligned}
& \left(\frac{\varphi_{0 i}(\stackrel{\mathrm{r}}{r}, \stackrel{\mathrm{r}}{\xi}, t)-\varphi_{e}(\stackrel{\mathrm{r}}{r}, \stackrel{\mathrm{r}}{\xi}, t)}{\tau_{e i}}\right)+\left(\frac{\varphi_{0 n}-\varphi_{e}}{\tau_{e n}}\right) \text { as } \varphi_{\alpha 0}=n_{\alpha}\left(2 \pi R T_{\alpha}\right)^{-\frac{3}{2}} \exp \left(\frac{-\left(\stackrel{\mathrm{r}}{\xi}-\stackrel{\mathbf{u}}{V}_{\alpha}\right)^{2}}{2 R T_{\alpha}}\right)
\end{aligned}
$$


The primary arguments $n_{\alpha}, \stackrel{\mathbf{u}}{V}$ and $T_{\alpha}$ that possess the character of the fundamental equations of motion can be obtained by taking moments of the kinetic equation for the distribution functions. As a result, the entire particles are reflected in full velocity accommodation from the plane plate, such that the plasma particles are reflected from the plane plate with its velocity. Thus, the boundary conditions for the plate velocity are:

$V_{x 2}(0, t)=V_{0} e^{-\Omega t}$ for $t>0$, as $V_{x}=V_{x 2}$ as $\xi_{y}>0$ and $V_{x}$ is finite as $\mathrm{y} \rightarrow \infty$ [27-28].

Substituting from Eqs. (1) and (2) inside Eq. (3) one gets;

(4) $\frac{\partial \varphi_{e}}{\partial t}+\xi_{y} \frac{\partial \varphi_{e}}{\partial y}-\frac{e B_{e z}}{m_{e} c}\left(\xi_{y} \frac{\partial \varphi_{e}}{\partial \xi_{x}}-\xi_{x} \frac{\partial \varphi_{e}}{\partial \xi_{y}}\right)+\frac{e E_{e x}}{m_{e}} \frac{\partial \varphi_{e}}{\partial \xi_{x}}=\left(\frac{\varphi_{0 e}-\varphi_{e}}{\tau_{e e}}\right)+\left(\frac{\varphi_{0 i}-\varphi_{e}}{\tau_{e i}}\right)+\left(\frac{\varphi_{0 n}-\varphi_{e}}{\tau_{e n}}\right)$,

as $\tau_{e e}, \tau_{e i}$ and $\tau_{e n}$ are electron-electron, electron-ion, and electron-neutral relaxation times respectively, that are acquired by [27, 31-32]:

(5) $\tau_{e e}=\left(\frac{3 \sqrt{m_{e}} K_{B}{ }^{\frac{3}{2}} T_{e}^{\frac{3}{2}}}{4 \sqrt{\pi} n_{e} e^{4} \log \left[\Lambda_{e e}\right]}\right), \tau_{e i}=\left(\frac{3 \sqrt{m_{e}} K_{B}{ }^{\frac{3}{2}} T_{e}^{\frac{3}{2}}}{4 \sqrt{2 \pi} n_{i} e^{4} Z^{2} \log \left[\Lambda_{e i}\right]}\right)$ and $\tau_{e n}=\left(\frac{3 m_{e} K_{B}{ }^{\frac{3}{2}} T_{e}^{\frac{3}{2}}}{4 \sqrt{\pi m_{n}} n_{e} e^{4} \log \left[\Lambda_{e n}\right]}\right)$

Here $\log [\Lambda]=\log \left[4 \pi n \lambda_{D}^{3}\right] \quad Z$ are the Coulomb Logarithm and the degree of ionization, respectively, and $\lambda_{D e}=\lambda_{D i}=\lambda_{D}$ is the Debye length.

The pattern of the cone of influence suggested by Lee's [32-33] for the solution of Boltzmann's kinetic equation is used. Let us write the solution of Eq. (4) in the form:

$$
\varphi=\left\{\begin{array}{ll}
\varphi_{1}=n(2 \pi R T)^{-\frac{3}{2}}\left(1+\frac{\xi_{x} V_{x 1}}{R T}\right) \exp \left(\frac{-\xi^{2}}{2 R T}\right) & \text { for } \xi_{\mathrm{y}}<0 \downarrow \\
\varphi_{2}=n(2 \pi R T)^{-\frac{3}{2}}\left(1+\frac{\xi_{x} V_{x 2}}{R T}\right) \exp \left(\frac{-\xi^{2}}{2 R T}\right) & \text { for } \xi_{\mathrm{y}}>0 \uparrow
\end{array},\right.
$$

here $V_{x 1}$ and $V_{x 2}$ are two underdetermined functions of time $t$ and the single space variable $y$. Utilizing Grad's moment method in Ref. [13] multiplying Eq. (4) by $\psi_{j}(\xi)$ and integrating overall values of $\stackrel{\boldsymbol{\xi}}{\boldsymbol{\xi}}$, we get the significant transfer equations for electrons in the following form:

$$
\begin{aligned}
& \frac{\partial}{\partial t} \int \psi_{j} \varphi_{e} d \underline{\xi}+\frac{\partial}{\partial y} \int \xi_{y} \psi_{j} \varphi_{e} d \underline{\xi}+\frac{e E_{x e}}{m_{e}} \int \varphi_{e} \frac{\partial \psi_{j}}{\partial \xi_{x}} d \underline{\xi}+-\frac{e B_{z e}}{m_{e} c} \int\left(\xi_{x} \frac{\partial \psi_{j}}{\partial \xi_{y}}-\xi_{y} \frac{\partial \psi_{j}}{\partial \xi_{x}}\right) d \underline{\xi} \\
& =v_{e e} \int\left(\varphi_{0 e}-\varphi_{e}\right) \psi_{j} d \underline{\xi}+v_{e i} \int\left(\varphi_{0 i}-\varphi_{e}\right) \psi_{j} d \underline{\xi}+v_{e n} \int\left(\varphi_{0 n}-\varphi_{e}\right) \psi_{j} d \underline{\xi}
\end{aligned}
$$

All integrals over the velocity dimension are evaluated using the following relations [6, 10],

(8) $\int \psi_{j}(\stackrel{\mathbf{u}}{\boldsymbol{C}}) \varphi d \underline{\xi}=\int_{-\infty}^{\infty} \int_{-\infty}^{0} \int_{-\infty}^{\infty} \psi_{j} \varphi_{1} d \underline{\xi}+\int_{-\infty}^{\infty} \int_{0}^{\infty} \int_{-\infty}^{\infty} \psi_{j} \varphi_{2} d \underline{\xi}$ as $\psi_{j}=\psi_{j}(\stackrel{\mathbf{\xi}}{\xi}), j=1,2$ and

$d \underline{\xi}=d \xi_{x} d \xi_{y} d \xi_{z}$, as $\xi_{x}, \xi_{y}$ and $\xi_{z}$ are the particle velocities components along $x, y$, and $z$ - axes, respectively. The electric and magnetic fields $E$ and $B$ can also get from Maxwell's equations, for electrons as follows:

(9) $\frac{\partial E_{x e}}{\partial y}-\frac{1}{c} \frac{\partial B_{z e}}{\partial t}=0$

(10) $\frac{\partial B_{z e}}{\partial y}-\frac{1}{c} \frac{\partial E_{x e}}{\partial t}-\frac{4 \pi e n_{e}}{c_{0}} V_{x e}=0$. 
Here $n=\int \varphi d \underline{\xi}, \quad n V_{x}=\int \xi_{x} \varphi d \underline{\xi}$, and the initial and boundary conditions:

$$
\left.\begin{array}{l}
E_{x}(y, 0)=0, B_{z}(y, 0)=B_{0} \\
E_{x}(y, t) \text { and } B_{z}(y, t) \text { are finite as } \mathrm{y} \rightarrow \infty .
\end{array}\right\}
$$

We offered the non-dimensional variables defined by:

$$
\left.\begin{array}{l}
t=t^{*} \tau_{e e}, y=y^{*}\left(\tau_{e e} c\right), V_{x}=V_{x}^{*} c, \tau_{x y}=\tau_{x y}^{*} c, M=\frac{V_{0}}{c}, E_{x}=E_{x}^{*}\left(\frac{m_{e} c}{e \tau_{e e}}\right), \rho=n m \\
B_{z}=B_{z}^{*}\left(\frac{m_{e} c}{e \tau_{e e}}\right), \gamma=\frac{m_{e}}{m_{i}}, d U=d U^{*}\left(K_{B} T_{e}\right) \text { and } \varphi_{j}=\varphi_{j}^{*} n_{e}\left(2 \pi R T_{e}\right)^{-\frac{3}{2}}, j=0,1,2
\end{array}\right\}
$$

The change in density and temperature are negligible for small values of Mach number, i.e., at $M^{2}=1$, therefore, we can assume that $n_{\alpha}=1+O\left(M^{2}\right)$ and $T_{\alpha}=1+O\left(M^{2}\right)$. Then, we can write the mean velocity and shear stress in the following form:

$$
V_{x}=\frac{1}{2}\left(V_{x 1}+V_{x 2}\right), \tau_{x y}=\frac{P_{x y}}{\rho V_{0} \sqrt{R T_{e} / 2 \pi}}=\left(V_{x 2}-V_{x 1}\right) .
$$

Here, $P_{x y}$ is defined by the relation $P_{x y}=m \int\left(\xi_{x}-V_{x}\right) \xi_{y} \phi d \underline{\xi}$ [32].

Utilizing the non-dimensional variable, Eq. (7) with $\psi_{1}=\xi_{x}$ and $\psi_{2}=\xi_{x} \xi_{y}$ becomes:

(14) $\frac{\partial V_{e x}^{*}}{\partial t^{*}}+\frac{\partial \tau_{e x y}^{*}}{\partial y^{*}}-E_{e x}^{*}=0$

(15) $\frac{\partial \tau_{e x y}^{*}}{\partial t^{*}}+2 \pi \frac{\partial V_{e x}^{*}}{\partial y^{*}}+\tau_{e x y}^{*}=0$.

Furthermore, the initial and boundary conditions take the form:

$$
\left.\begin{array}{c}
V_{e x}^{*}\left(y^{*}, 0\right)=\tau_{e x y}^{*}\left(y^{*}, 0\right)=0, \\
2 V_{e x}^{*}\left(0, t^{*}\right)+\tau_{e x}^{*}\left(0, t^{*}\right)=2 M e^{-\varepsilon t^{*}} \\
V_{e x}^{*} \text { and } \tau_{e x y}^{*} \text { are finite as } \mathrm{y} \rightarrow \infty, \varepsilon=w \tau_{e e} .
\end{array}\right\}
$$

Here and henceforth, for the sake of notational simplicity, we will drop the star on the nondimensional variables. Thus, we have the next system of equations representing the boundary value problem for electrons:

$$
\begin{aligned}
& \text { (17) } \frac{\partial V_{e x}}{\partial t}+\frac{\partial \tau_{e x y}}{\partial y}-E_{e x}=0, \\
& \text { (18) } \frac{\partial \tau_{e x y}}{\partial t}+2 \pi \frac{\partial V_{e x}}{\partial y}+\left(1+\frac{v_{e i}}{v_{e e}}+\frac{v_{e n}}{v_{e e}}\right) \tau_{e x y}=0, \\
& \text { (19) } \frac{\partial E_{e x}}{\partial y}-\frac{\partial B_{e z}}{\partial t}=0, \\
& \text { (20) } \frac{\partial B_{e z}}{\partial y}-\frac{\partial E_{e x}}{\partial t}-w_{e 0} V_{e x}=0, w_{e 0}=\left(\frac{n_{e} e^{2}}{m_{e} v_{e e}^{2}}\right) .
\end{aligned}
$$

Utilizing the traveling wave solution method [34-36] considering the new variable $\theta$ as:

(21) $\theta=k y-\omega t$.

This procedure will transform the dependent variables as functions of the new variable $\theta$. Also, the transformation constants $k$ and $\omega$ can be determined from the initial and boundary conditions, such that they do not depend on the properties of the plasma flow [35-36].

The partial derivatives of Eqs. (17)-(20) can be determined from Eq. (21) as follows: 
(22) $\frac{\partial}{\partial t}=-\omega \frac{\partial}{\partial \theta}, \frac{\partial}{\partial y}=k \frac{\partial}{\partial \theta}$ and $\frac{\partial^{n}}{\partial t^{n}}=(-1)^{n} \omega^{n} \frac{\partial^{n}}{\partial \theta^{n}}, \frac{\partial^{n}}{\partial y^{n}}=k^{n} \frac{\partial^{n}}{\partial \theta^{n}}$.

Substituting from Eqs. (21)-(22) into Eqs. (17)-(20). After various calculus manipulations, the obtained equations can be reduced to one equation:

$(23)\left(\left(\omega^{2}-k^{2}\right)\left(\frac{2 \pi k^{2}}{\omega}-\omega\right)\right) \frac{d^{3} V_{e x}(\theta)}{d \theta^{3}}+w_{c}\left(\omega^{2}-k^{2}\right) \frac{d^{2} V_{e x}(\theta)}{d \theta^{2}}+w_{e 0} \omega \frac{d V_{e x}(\theta)}{d \theta}+w_{e 0} w_{c} V_{e x}(\theta)=0$
$w_{c}=\left(1+\frac{v_{e i}}{v_{e e}}+\frac{v_{e n}}{v_{e e}}\right)$.
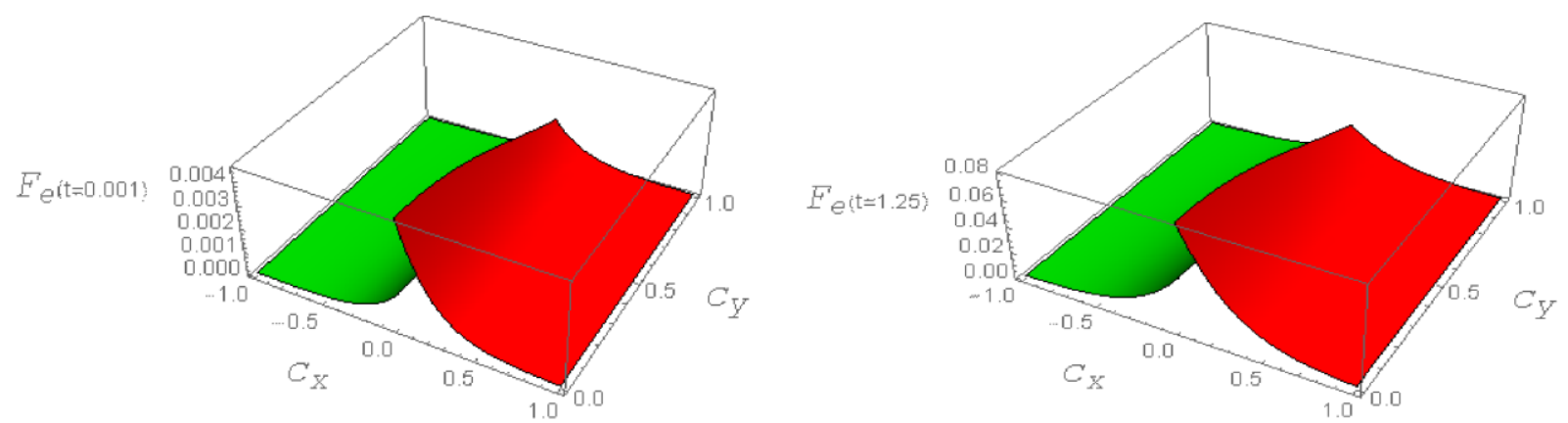

Fig. 1A. The perturbed electron velocity distribution functions $F_{e}\left[F_{1}\left(\right.\right.$ green), $F_{2}($ red $\left.)\right]$ at $(t=0.001$ and 1.25$)$ for a fixed $y$ value $(y=1)$ with the Mach number of the plate $M=0.01$.

Introducing the initial and boundary conditions after dropping the stars from the non-dimensional variables:

$$
\begin{aligned}
& E_{e x}(\theta=0)=\tau_{e x y}(\theta=0)=0, B_{e z}(\theta=0)=B_{0} \\
& 2 V_{e x}(\theta=-\omega)+\tau_{e x y}(\theta=-\omega)=2 M e^{-\varepsilon} \text { at } y=0, \text { e.g., } t=1 \\
& V_{e x}, \tau_{e x y}, E_{e x} \text { and } B_{e z} \text { are finite as } \theta \rightarrow-\infty .
\end{aligned}
$$

As a result of calculations on the model equations, we get the differential equation (23) with the conditions in Eqs. (24). This model equation is a third-order ordinary homogeneous differential equation that can be solved precisely with the help of any symbolic software using the initial and boundary conditions (24). Using these model solutions, a more elaborate description can be provided for the flows of laboratory argon plasma.

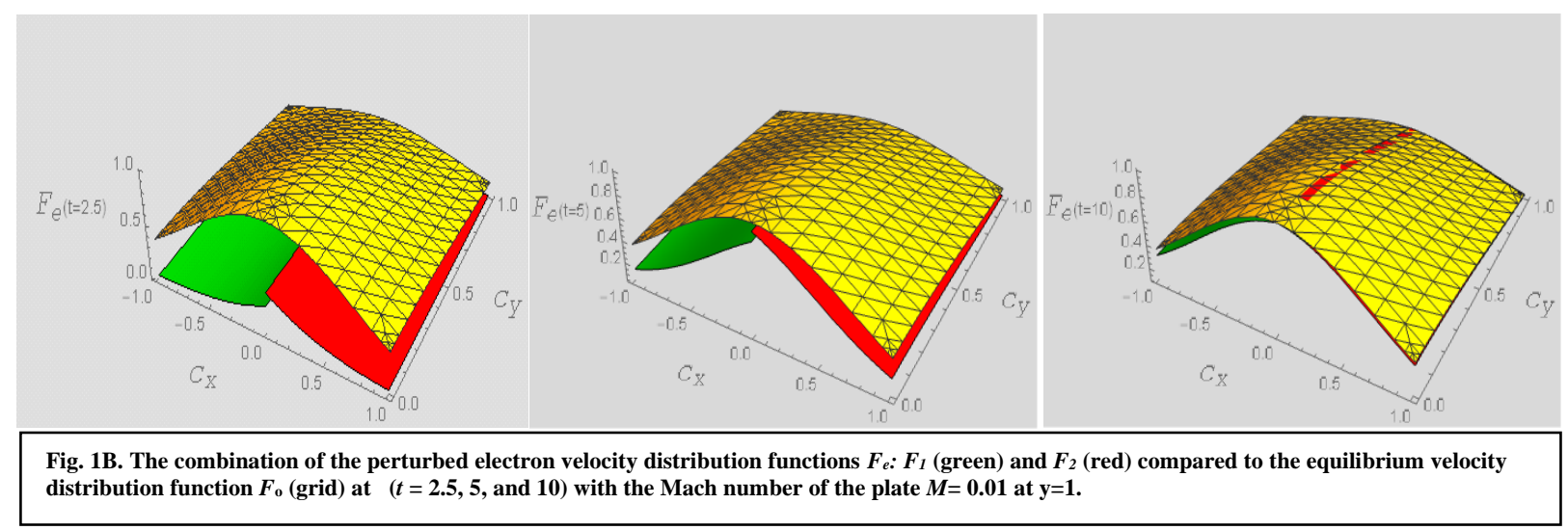




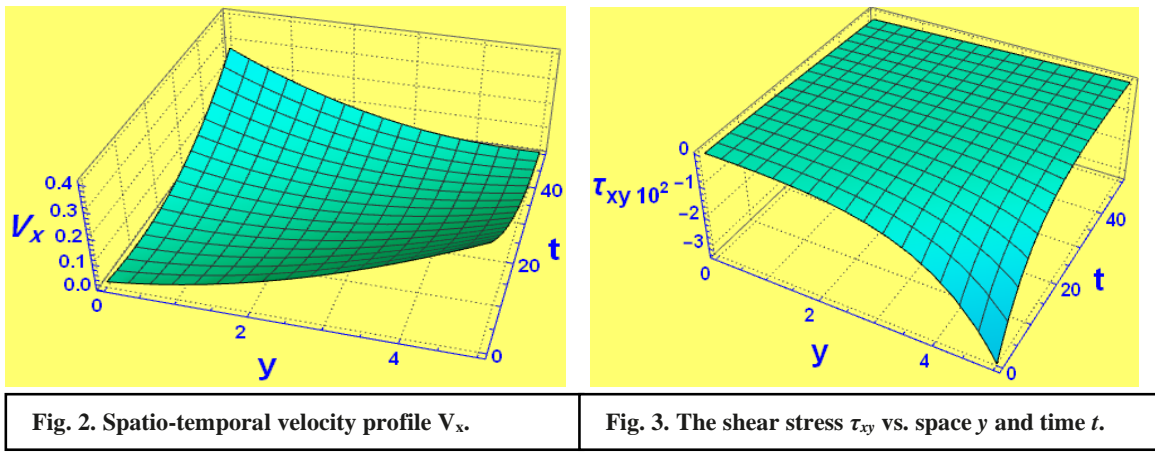

\section{The Non-Equilibrium Thermodynamic Investigations}

The problem of the non-equilibrium thermodynamics of irreversible processes is fundamental when modeling any gas flow, and it continues to present significant significance in the plasma dynamics. As a result of intensive research efforts in this field, scientists have found that the theoretical significance of that theory and its numerous applications in various branches of science starts from the thermodynamic laws and the essentials of the H-theorem. Now, we can evaluate the entropy per unit mass $S$ in a non-dimensional form as in the following relation; see, e.g., Ref. [18-21]:

$$
S=-\int \varphi_{e} \ln \varphi_{e} d \underline{\xi}=-\left(\int \varphi_{e 1} \ln \varphi_{e 1} d \underline{\xi}+\int \varphi_{e 2} \ln \varphi_{e 2} d \underline{\xi}\right)=-\pi^{\frac{3}{2}}\left[\left(V_{x 1}^{2}+V_{x 2}^{2}\right)-0.66\right] .
$$

As a consequence, we can get the entropy flux component in the $y$-direction:

$$
J_{y}{ }^{(S)}=-\int \xi_{y} \varphi_{e} \ln \varphi_{e} d \underline{\xi}=-\left(\int \xi_{y} \varphi_{e 1} \ln \varphi_{e 1} d \underline{\xi}+\int \xi_{y} \varphi_{e 2} \ln \varphi_{e 2} d \underline{\xi}\right)=\left[\pi\left(V_{x 1}{ }^{2}+V_{x 2}{ }^{2}\right)\right] .
$$

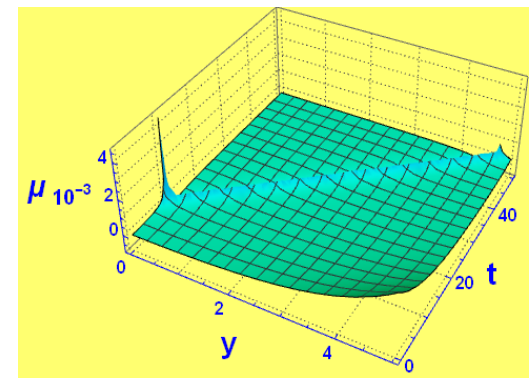

Fig. 4. The viscosity coefficient $\mu$ vs. space y and time $t$.

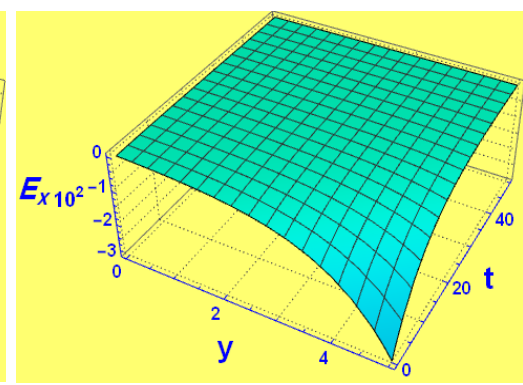

Fig. 5. The induced electric field $E_{x}$ vs. space $y$ and time $t$.

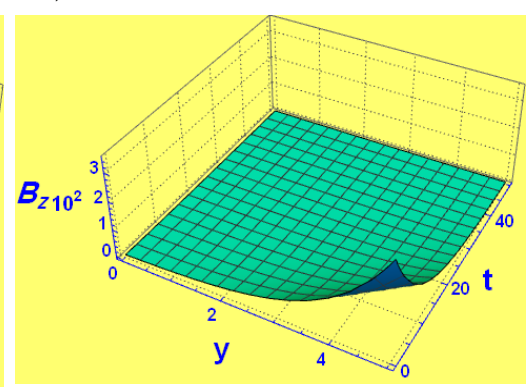

Fig. 6. The applied magnetic field $B_{z}$ vs. space $y$ and time $t$.

From the entropy balance relation, one can define the entropy production law in the local form:

$$
\sigma=\frac{\partial S}{\partial t}+\stackrel{\mathrm{r}}{\nabla} \bullet J^{(S)}
$$

Taking into account the electromagnetic field energy, we can investigate the internal energy change of the entire system using the extended Gibbs formula [37] that includes the entire energy balance. The electromagnetic energy is used to distinguish the plasma gas into paramagnetic and diamagnetic gas. It is well known that if the molecular orbital diagram contains unpaired electrons, the plasma is paramagnetic gas. In contrast, if the electrons are paired, the plasma is regarded as diamagnetic.

Now, we can use the $1^{\text {st }}$ law of thermodynamics to formulate the change in the total energy of the system for both kinds of magnetization, including the electromagnetic field energy balance as follows:

i) In the paramagnetic case: the internal energy change can be written in terms of the entropy, $S$, polarization, $P$ and specific magnetization, $M$, which are the thermodynamic coordinates due to 
the temperature, $T$, electric field, $E$ and magnetic field, $B$, respectively. As a consequence, the extensive three participants of the internal energy change in the Gibbs relation:

$d U=d U_{S}+d U_{p o l}+d U_{p a \alpha}$, with $d U_{S}=T d S$ is the internal energy change according to the change in entropy, $d U_{p o l}=E d P$ is the internal energy change according to change in polarization, and $d U_{\text {para }}=B d M$ is the internal energy change according to the change in magnetization, where $M$ is calculated from the equation [26-27,37]: $\frac{\partial S}{\partial M_{B}}=-\frac{B}{T} \Rightarrow M_{B}=-\int\left(\frac{T}{B} \frac{\partial S}{\partial y}\right) d y$.

Using the non-dimensional variables $U^{*}=\frac{U}{m_{e} c^{2}}, M_{B}^{*}=M_{B}\left(\frac{v_{e i}}{e c}\right), p^{*}=p\left(\frac{v_{e e}}{e c}\right)$ in the Gibbs relation, we can get (after dropping the star) $d U=d S_{e}+f_{1} E_{x} d p+f_{1} B_{z} d M_{B}$.

ii) In the diamagnetic case: the internal energy change can be written in terms of the extensive thermodynamic quantities $S, P$, and the induced magnetic field, $B$, which symbolize the thermodynamic coordinates due to the intensive quantities $T, E$, and $M_{B}$, respectively, thus we have three participants in the internal energy modified in the Gibbs formula acquired by

$$
d U=d U_{S}+d U_{p o l}+d U_{d i a}
$$

as $d U_{\text {dia }}=-M_{B} d B$ is the internal energy change according to the change in the generated magnetic field induction, as $M_{B}=T \frac{\partial S}{\partial B}$ [26-27,37]. In the present case, the internal energy $d U$, in non-dimensional form, is written in the compact form as: $d U=d S+E d p-M_{B} d B$, and $d S=\left(\frac{\partial S}{\partial r}\right) \delta y+\left(\frac{\partial S}{\partial t}\right) \delta t$.

\section{Discussion}

In the present study, we have studied the unsteady behavior of plasma gas based on the Boltzmann kinetic theory and irreversible thermodynamics using the Boltzmann equation's exact traveling wave solution with the exact value of electron-electron, electron-ion, and electron-atom collision frequencies in the BGK technique of the collision terms. The results are illustrated in figures for dilute electron argon gas, considering the motion of a rigid body in ionic belts in the upper atmosphere. It is shown that the computations due to the methods of solution are well performed using standardized data for electron gas in an argon plasma. The numerical results fit very well with the plasma laboratory $[28,31]$ in the following two cases: The paramagnetic medium, based on the ionizing potential applied to the atoms, the argon gas loses single electrons. In contrast, in the diamagnetic case, the argon gas loses electron pairs.

We present the exact solutions of the model equation using the idea of the shooting numerical calculation method to estimate the transformation constants $k=0.85, \omega=0.1$ and Mach number of the plate $M=1.0 \times 10^{-2}$ as presented in [17]. The numerical calculations are carried out for the problem according to the following plasma fluid properties and conditions:

Boltzmann constant, initial temperature, electrons concentration, and diameter of the argon atom are given by $k_{B}=1.3807 \times 10^{-16} \mathrm{erg} / \mathrm{K}, T_{0}=200 \mathrm{~K}, n_{e}=10^{17} \mathrm{~cm}^{-3}, d=3.84 \times 10^{-8} \mathrm{~cm}$. The electron rest mass and electron charge are given by $m_{e}=9.093 \times 10^{-28} \mathrm{gm}, e=4.810^{-10} \mathrm{esu}$, which are used to determine the dimensionless control parameter $\alpha_{0}=1.6 \times 10^{-12}$. Besides, the electronion, electron-electron, and electron-neutral atom collision frequencies values are 
$v_{e i}=1.46 \times 10^{15} \mathrm{Sec}^{-1} ; v_{e e}=1.033 \times 10^{15} \mathrm{Sec}^{-1} ;$ and $v_{e n}=1.199 \times 10^{4} \mathrm{Sec}^{-1}$, respectively. Finally, the plasma means free path $\lambda=\left(\sqrt{2} \pi n_{e} d^{2}\right)^{-1}=1.526 \times 10^{-3} \mathrm{~cm}$, which is large compared with one of the most fundamental properties, which is the electron Debye length $\lambda_{D_{e}}=\sqrt{\frac{K_{B} T_{0}}{4 \pi n_{e} e^{2}}}=3.086 \times 10^{-7} \mathrm{~cm}$.

The behavior of solutions that appears in Figs. 1A-1B reveals that the effect of the plate motion on the electron velocity distribution function is very significant. Figure 1B displays the perturbed electron velocity distribution function in the nearby area of the flat plate that suddenly set in motion with Mach number equals to 0.01 . As shown in Fig. 1B, the deviation from equilibrium decreasing with time as the system tries to reach an equilibrium state as expected from Le Chatelier's principle. Therefore, the electron velocity distribution function $F_{e}$ approach to equilibrium velocity distribution function $F_{0}$ as $t=10$, a result which strengthens the interpretation of the equilibrium principle. According to Le Chatelier, the position of equilibrium at a certain point in time for the perturbed electron velocity distribution functions $F_{1}$ and $F_{2}$ approach to the equilibrium distribution function $F_{0}$, which is of interest to our problem (see Figs. 1B).

Figures 2-6 show respectively the Spatio-temporal graphics of the velocity, shear stress, viscosity, applied magnetic field, and the induced electric field according to it. We see that near the moving plate, the mean velocity of the electrons in Fig. 2 has a value equals to Mach number $M=0.01$ of the flat plate as $(y, t)=(0,0)$. It decreases exponentially with time, which gives a good agreement with the results presented in [26,28]. The shear stress decreases with time, as shown in Fig. 3. The viscosity coefficient follows the equilibrium law by counteracting the change such that the system tends to equilibrium with increasing viscosity, as seen in Fig. 4. We shed light upon the behavior of the generated electric and applied magnetic fields in Figs. 5 and 6. They illustrate that the electric and magnetic fields are decreasing in magnitudes tend to zero value over time. That is due to our assumption of the applied external magnetic field and due to the dependency of the generated electric field to the external magnetic field as they connected by Maxwell's equations. Away from the plate, the generated electric field increases with time (see Fig. 5). However, the magnetic field has the maximum value at the beginning of time. Then it decreases nonlinearly towards zero for all $y$ values, as shown in Fig. 6. In such a way, the effects of the electromagnetic fields are dominant compared with dynamic effects in the plasma flow model.

From the results for velocity, density, and temperature, which were obtained from the solution of the mathematical model, we can demonstrate the nonlinear behavior of the entropy, as seen in Fig. 7. The entropy $S$ of the system increases for argon plasma with time, which gives a good agreement with the $2^{\text {nd }}$ law of thermodynamics [29]. Also, the entropy production $\sigma$ decreases once the system goes towards equilibrium till it reaches the state of equilibrium, i.e., $\sigma=0$ and $S$ is maximum (see Figs. 7 and 8). The entropy production of the plasma model satisfies the fundamental laws of thermodynamics and the Boltzmann $\mathrm{H}$-theorem as $\sigma \geq 0$ for all values of the position $y$ and the time $t$, as shown in Fig. 8. As shown in Fig. 9, the change in the internal energy due to the variation of entropy nonlinearly decreases with time and space. For the paramagnetic plasma cases of $d U_{p o l}=E d P$ and $d U_{\text {para }}=B d M$, the internal energy changes are plotted in Figs. 10 and 11 . We note that the internal energy change due to the polarization and magnetization is nonlinearly increased with time and space.

An investigation of the stability of the system requires to study the time-rate of change (time derivatives) of the internal energy $U\left(\lim _{\Delta t \rightarrow 0} \frac{\Delta U}{\Delta t}=\lim _{\Delta t \rightarrow 0} \frac{\Delta U_{S}}{\Delta t}+\lim _{\Delta t \rightarrow 0} \frac{\Delta U_{p o l}}{\Delta t}+\lim _{\Delta t \rightarrow 0} \frac{\Delta U_{p a r a}}{\Delta t}\right)$. The righthand side terms can be positive, negative, or null. The positive values indicate an increase in time 
of internal energy, whereas negative values indicate a decrease in internal energy. On the other side, if the sum of the terms is equal to null, then the rate of change in the internal energy of the system vanishes. Besides, the necessary and sufficient condition for the system to be in the equilibrium state is $\sigma \geq 0$ and $\frac{d U}{d t} \leq 0$, which indicates in Fig. 8-11. The electron gyro-frequency, which is the angular frequency of the circular motion of a charged particle in the plane perpendicular to the applied magnetic field, gradually decreases with time for argon plasma and increases away from the plate (see Fig. 12). Whereas, in the vicinity of the plate, the electrons Larmor or gyro-radius, which is the radius of the circular motion of an electron in the plane perpendicular to the applied magnetic field, has the maximum value for argon, and then it vanishes away from the vicinity of the plate as shown in Fig. 13.

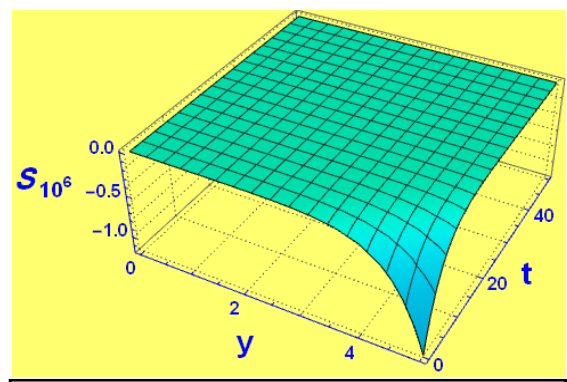

Fig. 7. The spatio-temporal entropy profile $S$.

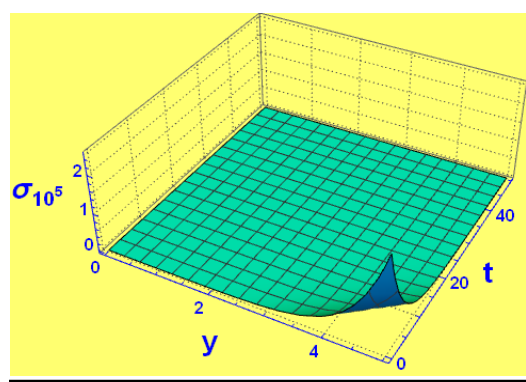

Fig. 8. The entropy production $\sigma$ vs. space $y$ and time $t$.

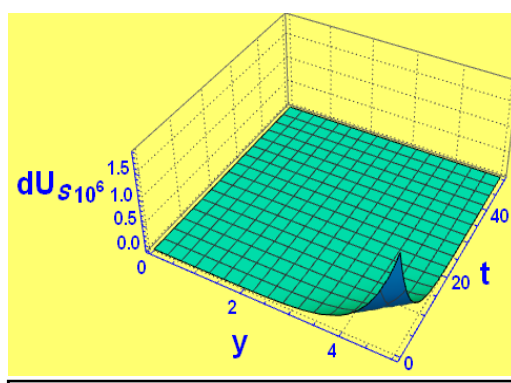

Fig. 9. The internal energy change $d U_{S}$ vs. space $y$ and time $t$.

\section{Conclusions}

In this paper, a detailed calculation of the effect of the collisions of the electrons with positive ions and neutral atoms operating with argon plasma has been made. Further, the current displacement term in the Maxwell equations is taken into consideration, which was ignored in the previous works; see $[17,18,20,26,28]$. We have elaborated a model to calculate the distribution functions, velocities, electromagnetic fields of the plasma flow under the effect of an applied magnetic field. Note that a good qualitative agreement was obtained between the obtained results and the laboratory results for argon plasma.

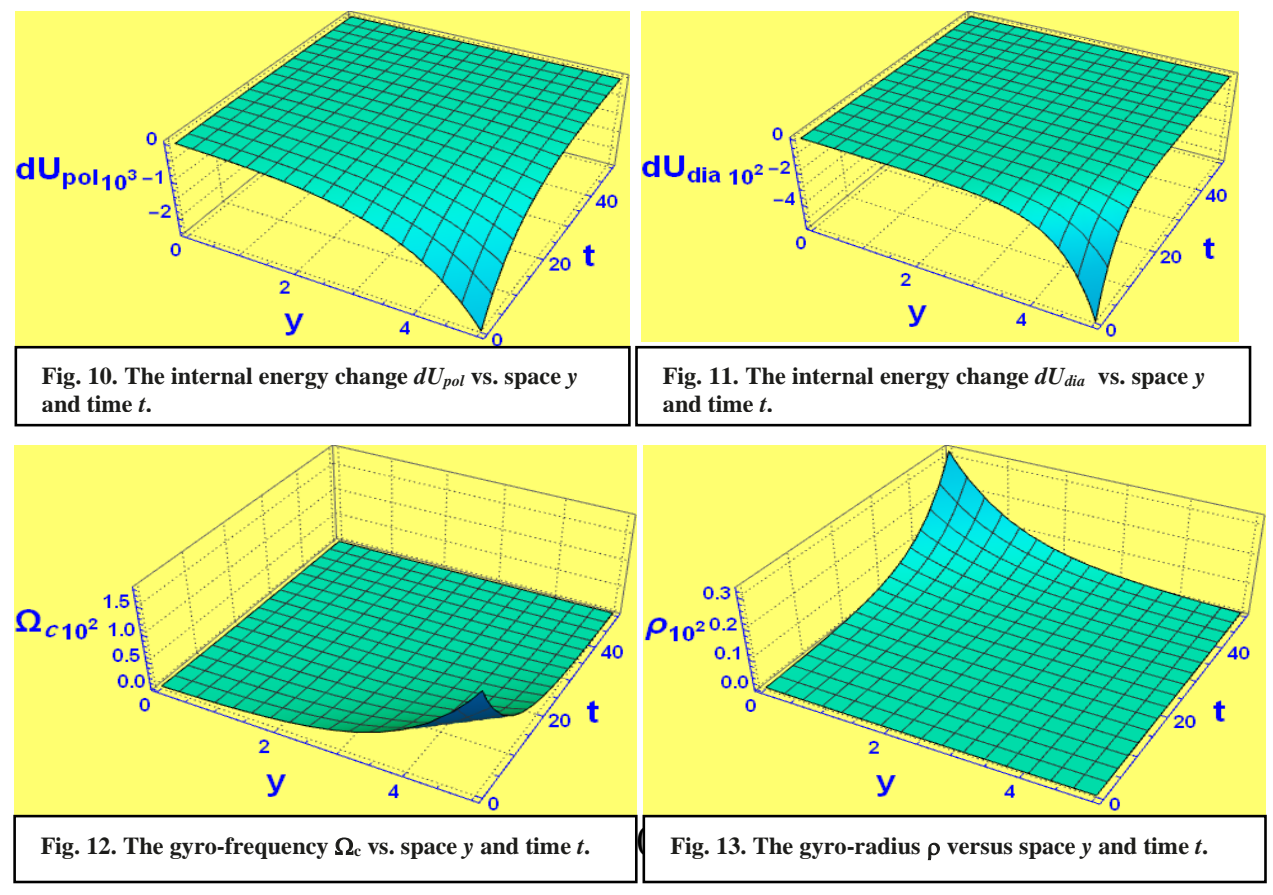


All graphics presented in the text have shown perfect agreement within the investigated ranges of the governing parameters and with initial and boundary conditions. This fact supports the following idea: Based on the BGK model, the Rayleigh flow problem can be studied in the framework of the Boltzmann kinetic equation supplemented by the Maxwell equations. Also, we can investigate the behavior of electron gas, which is generated from noble gases under the effect of a non-uniform unsteady external magnetic field using the method of moments of the kinetic equation for the two-sided distribution functions of the velocity. From the viewpoint of thermodynamics, the collisions between plasma components (ions, electrons, and atoms) are characterized by the fluctuation between loss and gain part of the energy of the charged particles (electrons) because of the interaction with the surroundings due to the plasma polarization and collisions around the state of equilibrium. Besides, the calculated distribution functions used to estimate entropy and entropy production that verify Boltzmann's H-theorem for non-equilibrium thermodynamic properties for the system under study. These properties are deeply discussed. After drawing the solutions, a good consistent between them was found with the thermodynamic laws. Finally, it should be added that the discovered behavior of the collision processes between electrons and ions in argon plasma based on the BGK technique of the Boltzmann kinetic equation might depend on the velocity of the moving plate in Rayleigh's problem of the rarefied gas dynamics and, further, this point has been examined minutely in this paper.

\section{References}

[1] C. G. N. Lee, K. J. Kanarik, and R. A. Gottscho, The grand challenges of plasma etching: a manufacturing perspective. J. Phys. D: Appl. Phys. 47: 273001, (2014).

[2] V. Miller, A. Lin, and A. Fridman, Why Target Immune Cells for Plasma Treatment of Cancer. Plasma Chem. Plasma Process, 36: 259-268 (2016).

[3] K.Weltmann, J. Kolb, M. Holub, D. Uhrlandt, M. Šimek, K. Ostrikov, S. Hamaguchi, U. Cvelbar, M. Černák, B. Locke, A. Fridman, P. Favia and K. Becker, The future for plasma science and technology. Plasma Processes and Polymers. 16:e1800118, (2019).

[4] G. Belmont, R. Grappin, F. Mottez, F. Pantellini, and G. Pelletier, Collisionless Plasmas in Astrophysics. WILEY-VCHVerlag GmbH \& Co. KGaA, Boschstr. 12, 69469, Weinheim, Germany, 2014.

[5] P. L. Bhatnagar, E. P. Gross, and M. Krook, A model for collision processes in gases. J. Phys. Rev. 94: 511-525 (1954).

[6] C. Cercignani, Slow Rarefied Flows: Theory and Application to Micro-Electro-Mechanical Systems. (Springer Science \& Business Media, 2006).

[7] M. H. Ernst and R. Brito, Anomalous velocity distributions in inelastic Maxwell gases. Nova science publishers, New York, pp.177-202 (2004).

[8] S. Chapman, On the kinetic theory of a gas. Part II. - A composite monatomic gas: Diffusion, viscosity, and thermal conduction, Phil. Trans. Royal Soc. London, 217A, 115,1916.

[9] D. Enskog, Kinetische Theorie der Vorgange in massing verdumten Gasen, Ph.D. Thesis, University of Uppsala, Sweden, 1917.

[10] S. Chapman and T. G. Cowling, The mathematical theory of non-uniform gases, (Cambridge university press, 1970). 
[11] H. Struchtrup, Grad's moment method. In: Macroscopic Transport Equations for Rarefied Gas Flows. Interaction of Mechanics and Mathematics. (Springer, Berlin, Heidelberg, 2005).

[12] Z. Donko and N. Dyatko, First-principles particle simulation and Boltzmann equation analysis of negative differential conductivity and transient negative mobility effects in xenon. Eur. Phys. J. D 70: 135 (2016).

[13] H. Grad, On the kinetic theory of rarefied gases. Comm. Pure Appl. Math. 2: 331-407 (1949). [14] A. M. Abourabia, and T. Z. Abdel Wahid, J. Non-Equilibrium Thermodynamics, 36 (2011), 75-98.

[15] A. M. Abourabia, and T. Z. Abdel Wahid, J. Non-Equilibrium Thermodynamics, 37(1): 1-25 (2012).

[16] A. M. Abourabia, and T. Z. Abdel Wahid, Can. J. Phys. 90: 137-149 (2012).

[17] T. Z. Abdel Wahid, J. Non-Equilibrium Thermodynamics, 37(2): 119-141 (2012).

[18] T. Z. Abdel Wahid and S.K. Elagan, Can. J. Phys. 90: 987-998 (2012).

[19] T. Z. Abdel Wahid, Can. J. Phys. 91(3): 201-210 (2013).

[20] T. Z. Abdel Wahid, Mathematical Problems in Engineering (ID 503729):1-13 (2013).

[21] T. Z. Abdel Wahid, Sohag Journal of Mathematics 2(2): 75-87 (2015).

[22] T. Z. Abdel Wahid, American Journal of Physics and Applications, 2(6): 121-134 (2014).

[23] W. Marques, A.R. Méndez, On the kinetic theory of vehicular traffic flow: Chapman-Enskog expansion versus Grad's moment method. Physica A. 392: 3430-3440 (2013).

[24] G. M. Kremer, An Introduction to the Boltzmann Equation and Transport Processes in Gases (Springer-Verlag Berlin Heidelberg, 2010).

[25] T. S. Chang and C. M. Chang, Rayleigh's problem in collisionless plasmas. Plasma Phys. 13:695, (1971).

[26] A. M. Abourabia and R. E. Tolba, Eur. Phys. J. Plus. 127(58):1-11 (2012).

[27] A.M. Abourabia and T.Z. Abdel Wahid, Can. J. Phys. 88: 501-511 (2010).

[28] T. Z. Abdel Wahid, Fluid Mechanics, 4(1): 27-37 (2018).

[29] G. Lebon, D. Jou, and J. Casas-Vàzquez, Understanding non-equilibrium thermodynamics: foundations, applications, frontiers. Springer-Verlag, Berlin, Heidelberg, Germany, (2008).

[30] T. Z. Abdel Wahid, The Effect of Lorentz and Centrifugal Forces on Gases and Plasma. (LAMBERT Academic Publishing, Germany, ISBN: 978-620-2-05504-8, 2017).

[31] J. D. Huba, NRL plasma formulary. Naval Research Laboratory, Washington, D.C., (2019). [32] S. I. Braginskii, Transport processes in a plasma. Reviews of Plasma Physics, Volume 1. Authorized translation from Russian by Herbert Lashinsky, University of Maryland, USA. Edited by M. A. Leontovich. Published by Consultants Bureau, New York, p.205 (1965).

[33] L. Lees, Kinetic theory description of rarefied gas flow. J. Soc. Ind. Appl. Math. 13: 278 (1965).

[34] A. M. Abourabia, A. M. Morad, Exact traveling wave solutions of the van der Waals normal form for fluidized granular matter. Physica A 437: 333-350 (2015).

[35] J. Gratton, S. M. Mahajan, and F. Minotti "Non-Newtonian Gravity Creeping Flow. International Centre for Theoretical Physics, Trieste (Italy), 1-17 (1988).

[36] G. Nugroho, A. M. S. Ali, and Z. A. Abdul Karim, Towards a new simple analytical formulation of Navier-Stokes Equations. World Acd. of Sci., Eng. And Tec. (2009).

[37] P. Van der Linde, Periodica Polytechnica Ser. Chem. Eng. 12: 97 (1998). 